\title{
Variação entoacional no Português do Brasil: uma análise fonológica do contorno nuclear em enunciados declarativos e interrogativos
}

\author{
Joelma Castelo \& Sónia Frota \\ CLUL - Universidade de Lisboa
}

In this paper we present a phonological analysis of the nuclear contour of declarative and interrogative utterances in Brazilian Portuguese, as spoken in 7 regions, along the Atlantic Coast - Paraíba, Sergipe, Bahia (North); Minas Gerais, Rio de Janeiro (Center), Santa Catarina and Rio Grande do Sul (South). Declaratives present a falling contour, with phonetic differences across varieties. As for interrogatives, more variation was found. Namely, two types of nuclear contours were observed: a rising contour in the North and a rising-falling in the Center-South. Unlike declaratives, the two interrogative patterns display a phonological continuum, in which the Center-Southern pattern spreads towards the North.

Keywords: intonation, declarative, yes-no question, intonational variation, Brazilian Portuguese.

Palavras-chave: declarativas, interrogativas totais, variação entoacional, Português do Brasil

\section{Introdução}

As diferenças fonológicas entre declarativas e interrogativas totais são objeto de extensiva análise no Português (Frota, 2000, 2002, 2014). Duas razões explicam o especial interesse nesse estudo: o isomorfismo sintático existente entre os dois tipos frásicos e variação desencadeada nos dois tipos frásicos. É reportado na literatura que as declarativas e, em maior escala, as interrogativas sofrem variação geográfica no Português e em muitas outras línguas como no Italiano e no Inglês (Grabe, 2004; Savino, 2012; Frota et al., 2015). Desse modo, é esperado que diferentes estratégias fonológicas sejam utilizadas entre as regiões para opor os dois tipos frásicos. O objetivo do presente artigo é propor uma análise fonológica do contorno nuclear em enunciados declarativos e interrogativos, tendo em conta o mapeamento de 7 variedades do Português do Brasil (PB) ao longo da Costa Atlântica: Paraíba, Sergipe, Bahia (Norte), Minas Gerais, Rio de Janeiro (Centro), Santa Catarina e Rio Grande do Sul (Sul).

Muitas discussões têm surgido acerca da abordagem da variação linguística dentro dos estudos formais. Conhecer um dado sistema linguístico implica em analisá-lo consoante as variações que esse sistema abrange ao longo de sua extensão territorial, não se restringindo às propriedades da variedade padrão (Barbies, 2009). A consideração das diversidades no sistema motivadas pelo espaço euclidiano (aspectos geográficos que unem ou separam populações) bem como pelo espaço social (a manipulação do espaço mediada pelas ações humanas) conduzem a um entendimento mais completo da gramática em estudo (Britain, 2007). Nesse sentido, as variedades do PB apresentam em suas marcas a herança do contato entre o Português e línguas

Textos Selecionados. XXX Encontro Nacional da Associação Portuguesa de Linguística, Braga, APL, 2015, pp. 113-131, ISBN 978-989-97440-4-2. 
africanas e indígenas (Lobo, 2003). O multilinguismo que caracteriza a implementação do Português como língua predominante no litoral do Brasil ao longo do século XVII pode ser dividia em dois grupos: uma língua geral do Norte, de base tupinambá, usada para o contato entre colonizadores e indígenas, expandida a partir do Maranhão, e uma língua geral do Centro, de base Tupininquim, usada para o contato entre bandeirantes (brancos e negros) e índios do interior do Brasil, expandida a partir de Minas Gerais e de São Paulo (Lobo, 2003).

A divisão dialetal proposta por Nascentes (1953) em subfalares do Norte (Amazônico e Nordestino) e subfalares do Sul (Fluminense, Mineiro, Sulista) está em conformidade com a divisão histórica dos grupos linguísticos estabelecidos na primeira fase da história linguística do Brasil. Estudos dialetais recentes (Silva, 2011; Silvestre, 2012), com base na análise fonética de dados do projeto Atlas Linguístico do Brasil em 25 capitais brasileiras revelam a existência de três grupos dialetais - Norte, Centro e Sul para as declarativas e dois grupos Norte e Centro-Sul, para as interrogativas. Esses estudos carecem, no entanto, de uma análise fonológica dos padrões entoacionais encontrados em cada região. Considerando, de um lado, a diversidade linguística que caracteriza a dimensão geográfica do $\mathrm{PB}$ e, de outro, a uniformidade que caracteriza seu sistema, a contribuição do presente estudo consiste em descrever e analisar o comportamento entoacional das declarativas e interrogativas, com o objetivo de propor uma análise fonológica uniforme que consiga explicar as regras subjacentes à realização de uma grande diversidade de contornos encontrados na superfície dos enunciados, bem como as estratégias de oposição entre os tipos frásicos declarativo e interrogativo total nas variedades do PB.

O presente trabalho está dividido nas seguintes partes: após essa introdução, abordamos na seção 2 alguns aspectos sintático-morfológicos (2.1) e entoacionais (2.2) que distinguem enunciados declarativos e interrogativos totais. Na seção 3, é feita a descrição do corpus e metodologia adotados no estudo. $\mathrm{Na}$ seção 4 , são descritos e discutidos os resultados do estudo, tendo como base as análises acústicas e estatísticas dos dados e, por fim, os resultados são interpretados consoante o principal objetivo do estudo: propor uma análise fonológica para os padrões entoacionais apresentados na seção 5. As conclusões do estudo são apresentadas na seção 6.

\section{Aspectos da gramática dos tipos frásicos declarativos e interrogativos}

\subsection{Aspectos não-entoacionais}

Os tipos frásicos declarativo e interrogativo total podem ser caracterizados por diferentes propriedades linguísticas (sintáticas, morfo-sintáticas, prosódicas e lexicais), que são empregadas para expressar as intenções comunicativas do locutor (Mateus et al., 2003). Apesar dos muitos tipos pragmáticos de declarativas e sobretudo de perguntas, a divisão em enunciados neutros e não-neutros leva em consideração a pressuposição informacional ou situacional. Os tipos frásicos abordados no presente estudo são produzidos em contextos pragmáticos distintos. As perguntas neutras são enunciadas quando o sujeito quer saber uma informação que desconhece completamente. Por isso, 
conta com a colaboração do seu interlocutor para obter a informação de que precisa. Esse tipo de pergunta é enunciado, por exemplo, numa situação em que o sujeito entra numa loja e tem a intenção de saber se são vendidos doces naquele estabelecimento, produzindo o enunciado "Vocês vendem doces?". A declarativa neutra, por sua vez, é enunciada quando o sujeito tem a intenção de partilhar um valor de verdade com seu interlocutor, tal como poderia ocorrer na situação acima, em que o vendedor, em resposta à pergunta feita pelo cliente, responderia com a declarativa: "Sim, vendemos".

Assim como as especificidades semântico-pragmáticas dos tipos de enunciados não-neutros podem ser sintaticamente marcados nas línguas, como, por exemplo, através de estruturas clivadas empregadas para expressar o foco informacional em Português (Mateus et al., 2003) ou através do emprego de uma partícula gramatical específica na periferia esquerda da frase para expressar perguntas retóricas e antiexpectativas no dialeto falado na província de Belluno ao norte da Itália (Obanauer, 2006), os enunciados neutros declarativos e interrogativos podem apresentar marcas morfossintáticas que os diferenciem. Em línguas como o Inglês e o Alemão, por exemplo, as interrogativas neutras são divididas em dois grupos: as interrogative declaratives, que apresentam a mesma forma sintática das declarativas e as yes-no question, as quais desencadeiam alguma mudança sintática para expressar seu valor de interrogatividade, seja através de um marcador interrogativo seja através da inversão (Grabe, 2004; Haan, 2002). Diferentemente dessas línguas, o Português apresenta isomorfismo sintático entre declarativas e interrogativas neutras, por conseguinte, o uso de entidades fonológicas distintas configura-se como um importante recurso para marcar o contraste entre os dois tipos frásicos nessa língua.

\subsection{Aspectos entoacionais}

A entoação modal tem sido alvo de muitas pesquisas que pretendem comparar os sistemas fonológicos das línguas (Jun, 2005, 2014; Frota et al., 2015). Esses estudos mostram que as diferenças na superfície dos enunciados refletem uma organização fonológica subjacente empregada para expressar significados que estão além do nível da palavra (Gussenhoven, 2004; Ladd, 2008). É interessante observar que certos usos da frequência fundamental da voz humana podem ser gramaticalizados em conformidade com os usos primitivos da relação do homem com seu meio. O emprego de uma voz aguda pode ser considerada como sinônimo de alguma relação de subordinação seja através da sua condição biológica (crianças têm voz aguda quando comparadas a dos adultos), pragmática (fazemos pedidos com uma altura global da voz mais elevada) e até os tipos frásicos (frases inacabadas são geralmente produzidas com contornos ascendentes). A tendência da elevação global da altura melódica da voz ou da produção de uma melodia ascendente são sistematicamente empregadas para expressar interrogatividade. Sendo assim, os falantes fazem uso desse recurso para marcar seu grau de incerteza assim como a necessidade da ajuda do seu interlocutor para obter a informação de que necessita (Gussenhoven, 2004).

Diferentes tipos de estratégias entoacionais podem ser empregadas para expressar a modalidade frásica nas línguas. No Sueco, por exemplo, as mudanças no registro tonal são mais relevantes na diferenciação dos tipos frásicos, o que implica numa maior sensibilidade dos seus falantes na identificação de perguntas através de mudanças na 
gama de variação da F0, comparados aos falantes nativos do inglês americano, que são mais sensíveis às inflexões graduais da curva melódica. Esses exemplos demonstram como a forma fonética é empregada de forma seletiva entre as línguas, havendo uma relação arbitrária entre elas para definir a relação entre forma e significados frásicos e pragmáticos (Gussenhoven, 2002, 2004; Frota, submetido).

Estudos revelam que a principal distinção entre declarativas e interrogativas nas línguas românicas é desempenhada pelo contorno nuclear do sintagma entoacional (Frota \& Prieto, 2015). Em relação a esse traço, as línguas românicas aqui representadas podem ser divididas em dois grupos. O primeiro grupo que apresenta contraste marcado pelo núcleo do IP, o qual é formado por um contorno descendente (HL) nas declarativas e ascendente ( $\mathrm{LH})$ nas interrogativas, sendo ambas seguidas de uma fronteira baixa, como é o caso das variedades do sul do Italiano (Nápoles, Bari e Palermo) e do Espanhol Argentino (Grice et al., 2005; Prieto \& Roseano, 2010). O segundo grupo apresenta contraste marcado pela fronteira do IP: fronteira baixa (L\%) nas declarativas, normalmente precedida de um contorno nuclear baixo (L) ou descendente (HL); fronteira ascendente $(\mathrm{H} \%, \mathrm{HH} \%$ ou $\mathrm{LH} \%)$ nas interrogativas, normalmente precedida por um núcleo baixo (L), como é o caso do Espanhol, do Valenciano e de algumas variedades do Espanhol falado na América Latina (Equador Andino e México).

Estudos desenvolvidos para o Português Europeu (PE), com base na variedade urbana de Lisboa (Frota, 2002) e para o Português do Brasil, com base na variedade urbana do Rio de Janeiro (Moraes, 2008) mostram como o contraste fonológico entre declarativas e interrogativas pode ser marcado através de diferentes estratégias no Português. No PE, o contraste é marcado na fronteira do IP (L\% para declarativas e LH\% para interrogativas), sendo ambos os tipos frásicos precedidos por um acento descendente $\mathrm{H}+\mathrm{L}^{*}$. No $\mathrm{PB}$, por outro lado, o contraste entre os dois tipos frásicos é desempenhado pelo núcleo $\left(\mathrm{H}+\mathrm{L}^{*}\right.$ nas declarativas e $\mathrm{L}+\mathrm{H}$ nas interrogativas), seguido de fronteira baixa L\% (Frota \& Moraes, aceito). Descrições recentes baseadas em corpora de leitura e fala semi-espontânea encontram pouca variabilidade nas declarativas (Frota et al., 2015), sendo o contorno predominante $\mathrm{H}+\mathrm{L} * \mathrm{~L} \%$ comum para a maior parte das variedades (à exceção do Alentejo, que apresenta o contorno L* L\% na fala semi-espontânea), e grande variação nas interrogativas (Crespo-Sendra et al., 2014; Frota et al., 2015) tanto nas variedades do PE, quanto nas variedades do PB. As especificações fonológicas encontradas indicam que a oposição declarativas versus interrogativas pode ser desempenhada pelo núcleo, pela fronteira, ou por todo o contorno, apresentando, assim, um sistema de contraste mais complexo do que foi encontrado nas variedades-padrão de Lisboa e Rio de Janeiro.

\section{Metodologia}

\section{Corpus}

O corpus analisado é proveniente dos materiais do Projeto Atlas Interactivo da Prosódia do Português - InAPoP (Frota coord., 2012-2015), do qual foram selecionadas 11 frases declarativas (Frota, 2000) e 49 frases interrogativas totais (Frota, 2002; Silva, em curso). $\mathrm{O}$ corpus apresenta sentenças iguais para declarativas e interrogativas, o que permite uma comparação equilibrada entre os dois tipos frásicos. A variação da posição 
do acento lexical da última palavra prosódica (PW) foi sistematicamente controlada a fim de analisar a consistência do alinhamento temporal dos tons à fiada segmental, conforme observado nos exemplos abaixo.

\begin{tabular}{|c|l|}
\hline Frase & Constituição métrica da PW nuclear \\
\hline Ela foi ver (o André? & Palavra oxítona com 2 sílabas pretônicas \\
\hline $\begin{array}{c}\text { Ela vai ver (a } \\
\text { Mariana? }\end{array}$ Palavra paroxítona com 2 sílabas pretônicas \\
$\begin{array}{c}\text { Ela foi ver (a } \\
\text { Débora? }\end{array}$ & Palavra proparoxítona com 1 sílaba pretônicas \\
\hline $\begin{array}{c}\text { Ela vai ter (uma } \\
\text { gramática? }\end{array}$ & Palavra paroxítona com 3 sílabas pretônicas \\
\hline
\end{tabular}

\section{Participantes e regiões}

Os participantes da presente pesquisa foram entrevistados in locu em sete capitais brasileiras ao longo da Costa Atlântica: João Pessoa, na Paraíba (PBA); Aracaju, no Sergipe (SE) e Salvador, na Bahia (BA), ao Norte; Rio de Janeiro, no Rio de Janeiro (RJ), Belo Horizonte, em Minas Gerais (MG), ao Centro; Florianópolis, em Santa Catarina (SC) e Porto Alegre, no Rio Grande do Sul (RGS), ao Sul. Em cada localidade, foram entrevistadas 4 mulheres escolarizadas, entre 20 e 45 anos, nascidas e criadas na região.

\section{Análise}

Do corpus de leitura, foram selecionados um total de 480 enunciados em cada variedade, sendo 88 enunciados declarativos (11 sentenças x 2 repetições $\mathrm{x} 4$ informantes) e 392 interrogativas (49 sentenças x 2 repetições x 4 informantes). A análise acústica e perceptiva dos dados segue o sistema de anotação ToBI (Tone and Break Indices), desenvolvido para o Português (P-ToBI - Frota, 2014; Frota et al. 2015). Os dados foram ouvidos e analisados acusticamente através de três camadas de anotação (tonal, ortográfica e de fronteiras), criadas a partir do programa Praat (Boersma \& Weenink, 2010). Todos os dados foram analisados por um anotador e confirmados por um segundo anotador e uma parte das interrogativas foi previamente analisada por um terceiro anotador (Crespo-Sendra et al. 2014).

\section{Resultados}

\subsection{A homogeneidade do padrão declarativo}

Os enunciados declarativos apresentam um contorno nuclear descendente comum a todas as variedades analisadas. Esse contorno é formado por um acento nuclear descendente $\left(\mathrm{H}+\mathrm{L}^{*}\right)$ - com um tom baixo associado à sílaba tônica da última palavra prosódica do IP, precedido por um tom alto alinhado com a sílaba pretônica imediatamente anterior à tônica, e seguido de um tom de fronteira baixo (L\%), 
associado à fronteira do IP (vide fig. 1). Esse tipo de comportamento é consistente independentemente da posição do acento ou da distância entre a posição nuclear da palavra prosódica e as suas fronteiras direita e esquerda.

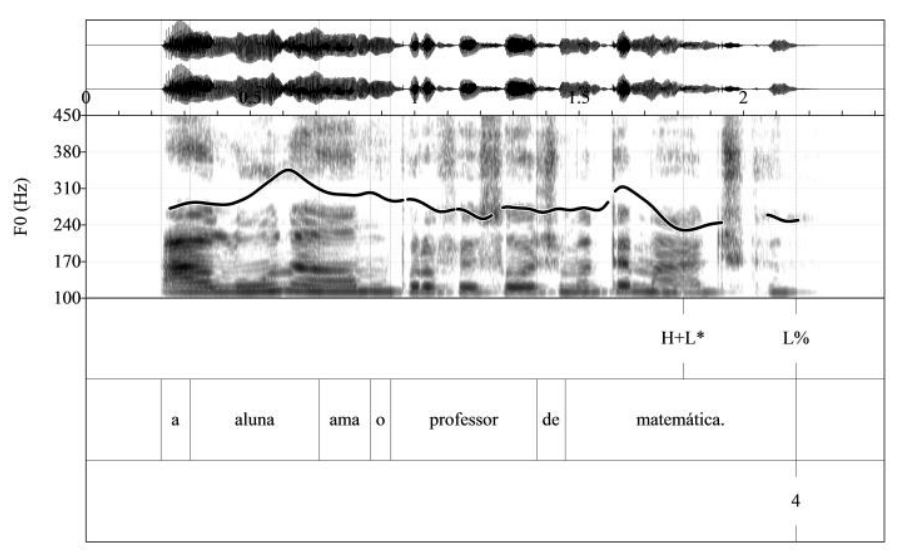

Figura 1: $\mathrm{O}$ contorno nuclear $\mathrm{H}+\mathrm{L} * \mathrm{~L} \%$, no enunciado declarativo "A aluna ama o professor de matemática.", produzido pela informante VP de MG.

Foram observadas ligeiras diferenças no alinhamento do tom baixo do núcleo entre as regiões. Nas variedades da Paraíba (PBA) e da Bahia (Bh), no Norte, o tom baixo ocupa a maior parte da sílaba tônica, até mesmo em enunciados com proparoxítonas finais. A anotação fonética $(\mathrm{H}+) \mathrm{L}^{*} \mathrm{~L} \%$ foi usada para indicar esse adiantamento do tom alto na pretônica, o qual implica na realização de movimento descendente no domínio dessa mesma sílaba, seguido de um tom baixo que ocupa a maior parte da tônica (vide fig. 2).

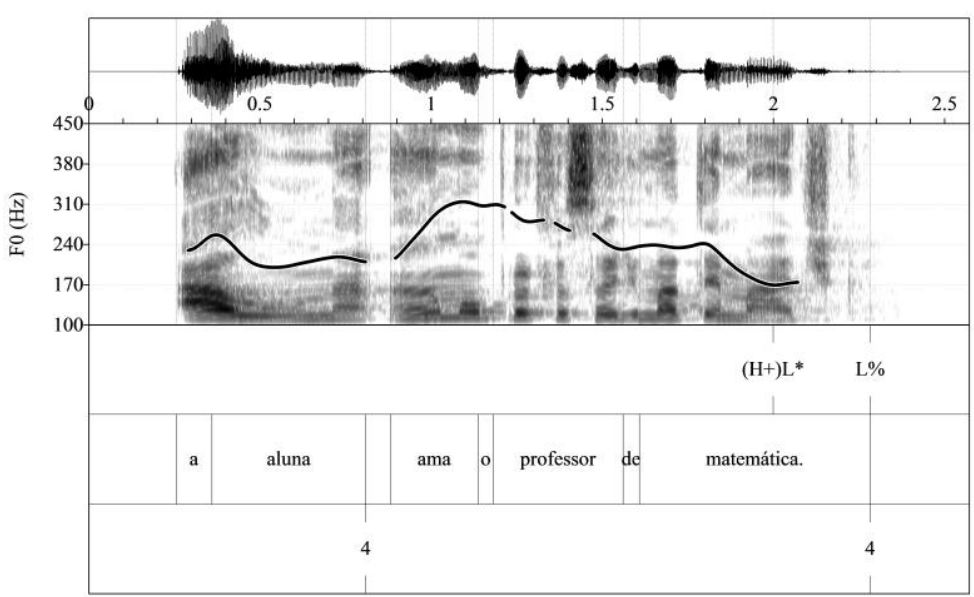

Figure 2: $\mathrm{O}$ contorno nuclear $(\mathrm{H}+) \mathrm{L}$ * $\mathrm{L} \%$ no enunciado declarativo "A aluna ama o professor de matemática.", produzido pela informante MA de Bh. 
$\mathrm{Na}$ tabela abaixo são apresentadas as porcentagens dos contornos declarativos observados entre as regiões, tendo em conta as diferenças em termos de alinhamento dos tons. $\mathrm{O}$ contorno $\mathrm{H}+\mathrm{L} * \mathrm{~L} \%$ é predominante em todas as regiões (acima de $80 \%$ das ocorrências no Centro e Sul e acima de $60 \%$ das ocorrências no Norte). exceto na Bahia, onde a presença do contorno $(\mathrm{H}+) \mathrm{L} * \mathrm{~L} \%$ foi predominante (63\% das ocorrências). Os resultados encontrados confirmam descrições anteriores (Silvestre, 2012; Frota et al., 2015; Frota \& Moraes, aceito). O contorno descendente declarativo do PB é o padrão entoacional da maioria das línguas românicas estudadas até hoje, como, por exemplo, o Italiano, o Espanhol, o Catalão e o PE (Grice, 2005; Prieto \& Roseano, 2010; Frota et al., 2015). À semelhança do que ocorre no PB, há uma grande homogeneidade do contorno $\mathrm{H}+\mathrm{L}^{*} \mathrm{~L} \%$ nas variedades faladas no sul da Itália, onde esse contorno é praticamente o único padrão descrito.

\begin{tabular}{|l|r|r|r|r|r|r|r|}
\hline \multicolumn{9}{|c|}{ Todas as regiões } \\
\hline & \multicolumn{3}{|c|}{ Norte } & \multicolumn{2}{c|}{ Centro } & \multicolumn{2}{c|}{ Sul } \\
\hline & PBA & SE & Bh & MG & RJ & SC & RGS \\
\hline$\left(\mathrm{H}+\mathrm{L}^{*} \mathrm{~L} \%\right.$ & $20 \%$ & $0 \%$ & $63 \%$ & $2 \%$ & $0 \%$ & $0 \%$ & $0 \%$ \\
\hline $\mathrm{H}+\mathrm{L} * \mathrm{~L} \%$ & $64 \%$ & $83 \%$ & $35 \%$ & $89 \%$ & $94 \%$ & $96 \%$ & $98 \%$ \\
\hline $\mathrm{L} * \mathrm{~L} \%$ & $16 \%$ & $17 \%$ & $2 \%$ & $8 \%$ & $6 \%$ & $4 \%$ & $2 \%$ \\
\hline
\end{tabular}

Tabela 1: Percentagens dos tipos de contornos nucleares das declarativas neutras.

As diferenças no alinhamento dos tons observadas entre Norte, Centro e Sul conduziram a uma análise estatística, a fim de conhecer a distribuição espacial da variação do padrão $\mathrm{H}+\mathrm{L}^{*} \mathrm{~L} \%$, em termos de alinhamento. Foram medidas as distâncias temporais do tom $\mathrm{H}$ em relação ao início (onset) da tônica e do tom $\mathrm{L}$ em relação ao final (offset) da tônica. Foi empregado o teste não paramétrico Mann-Whitney U, o qual compara aos pares as regiões Norte, Centro e Sul. Os testes confirmam as diferenças fonéticas no alinhamento dos tons $L$ na região Sul, a qual se comporta significativamente diferente do Centro $(Z=-8,939$ e $p<0,05)$ e do Norte $(Z=-9,433$ e $p$ $<0,05)$. Em relação ao alinhamento do tom $\mathrm{H}$, por outro lado, a oposição entre Norte e Sul é significativamente relevante $(Z=-2,462$ e $\mathrm{p}<0,05)$, no entanto o alinhamento do Norte não difere significativamente do Centro $(Z=-1,028$ e $p>0,05)$. Os resultados revelam uma clara oposição entre Norte e Sul, além de sugerir uma mudança gradual no contorno, constatada através dos resultados do alinhamento do tom $\mathrm{H}$, que exibe padrões misturados.

As diferenças no alinhamento do contorno descendente declarativo não apresentam pistas para uma oposição fonológica entre os tipos frásicos. Embora apresentem diferenças fonéticas no alinhamento, ora aproximando-se do onset ora do offset da tônica, os tons alto e baixo não ultrapassam os seguintes limites de ancoragem: a sílaba pretônica para o tom alto e a sílaba tônica para o tom baixo. Esse tipo de comportamento indica que as diferenças observadas no alinhamento dos tons apresentam uma única estrutura profunda $\mathrm{H}+\mathrm{L} * \mathrm{~L} \%$, a qual exibe uma gradual variação fonética no alinhamento dos tons, observada sobretudo no alinhamento do tom alto. Sendo assim, os extremos fonéticos coincidem com os extremos geográficos, sendo 
observada uma maior distância no alinhamento do Norte em relação ao Sul. Semelhante tipo de variação temporal também foi descrito para variedades do Norte e do Sul da Alemanha (Atterer \& Ladd, 2004): nas variedades do Norte, foi observado um alinhamento mais adiantado em relação à variedade do Sul. Assim como observado para o PB, as diferenças no alinhamento dos tons exibem pequenas distâncias temporais, a qual sugere uma variação gradual no alinhamento entre as variedades.

\section{2 A heterogeneidade do contorno nuclear interrogativo}

Os enunciados interrogativos no PB caracterizam-se por um contorno nuclear ascendente-descendente (LHL). Esse tipo de contorno varia de acordo com a região, sendo predominante no Centro e Sul e alternativo no Norte, onde ocorre predominantemente o contorno ascendente (LH). As variações na especificação fonológica em cada uma dessas configurações são descritas nas seções a seguir.

\subsubsection{O contorno nuclear ascendente do Norte}

O contorno nuclear ascendente apresenta propriedades fonológicas diferentes nas variedades do Norte, podendo ser formado por um núcleo baixo seguido de fronteira alta ( $\left.\mathrm{L}^{*} \mathrm{H} \%\right)$, predominante nas variedades da Paraíba e do Sergipe ou por um núcleo ascendente seguido de uma fronteira alta $\left(\mathrm{L}^{*}+\mathrm{H} \mathrm{H} \%\right)$, alternativo na variedade da Bahia. Embora os contornos nucleares $\mathrm{L}^{*} \mathrm{H} \%$ e $\mathrm{L}^{*}+\mathrm{H} \mathrm{H} \%$ apresentem o mesmo tipo de configuração, as diferenças em relação ao alinhamento dos tons à fiada segmental revelam estruturas profundas distintas. Ao passo que os sintagmas entoacionais (IP) formados pelo núcleo $\mathrm{L}^{*}$ exibem a subida para fronteira após a tônica (fig. 3), em IP's formados pelo núcleo $\mathrm{L}^{*}+\mathrm{H}$ essa subida decorre ao longo da vogal tônica, independentemente do número de postônicas (fig. 4). O comportamento dos eventos tonais em contexto proparoxítono final revela fortes evidências para a fronteira monotonal $\mathrm{H} \%$. O número de postônicas que separa o núcleo da periferia direita do IP não altera o ponto de subida de F0, que é sempre iniciada a partir do final da tônica, diferentemente do que ocorre com a variedade padrão do PE, por exemplo, nesses contextos, em que a subida final começa na última sílaba postônica devido a presença de uma fronteira bitonal do tipo $\mathrm{LH} \%$.

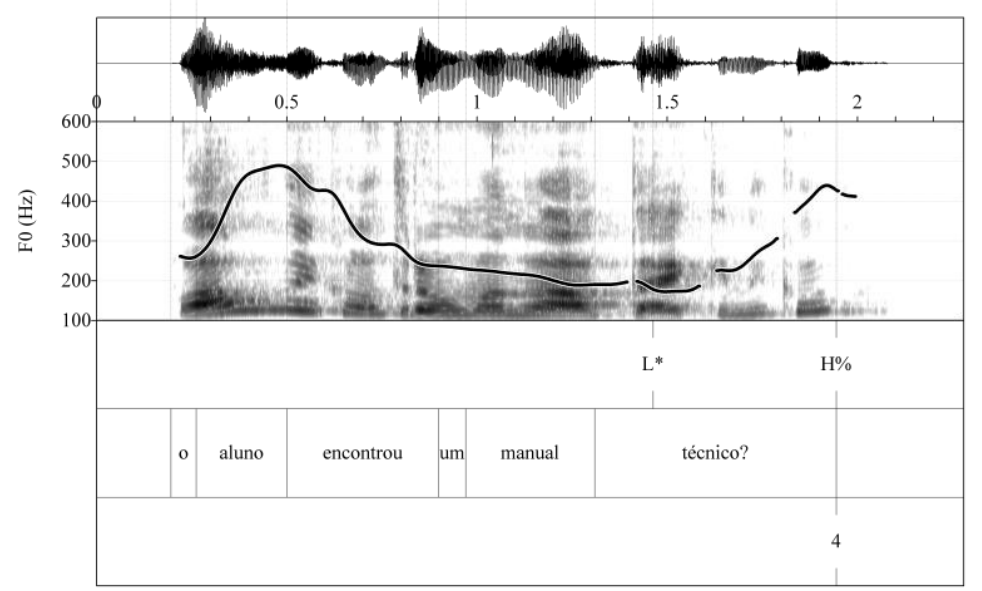

Figura 3: Enunciado interrogativo produzido pela informante KS de PBA. 


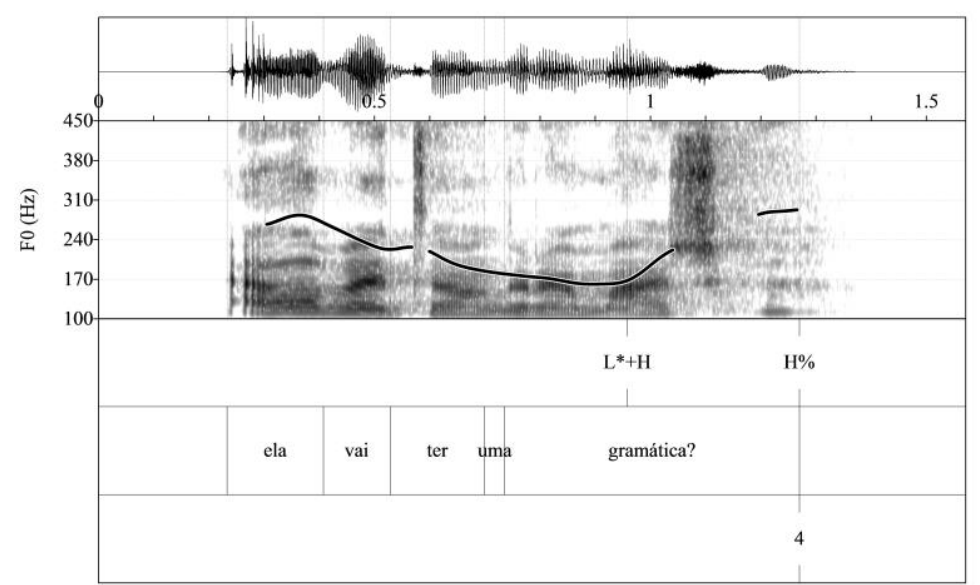

Figura 4: Enunciado interrogativo produzido pela informante MA de Bh.

A análise desses resultados mostra que a frequência do contorno principal nas variedades do Norte aponta para um continuum em termos de configuração e alinhamento dos contornos LH e LHL. Observa-se, na tabela 2, que a frequência do contorno LH do tipo L* H\% é decrescente entre PBA, SE e Bh (73\%, 44\% e 0\%). Nesta última variedade, o contorno $\mathrm{L}^{*} \mathrm{H} \%$ deixa de existir, sendo observada, em contrapartida, a presença do contorno ascendente com núcleo bitonal $\mathrm{L}^{*}+\mathrm{H} \mathrm{H} \%$ (18\% das ocorrências). As frequências dos contornos secundários também apontam para presença de um continuum no contorno LHL. O contorno secundário L* HL\% nas variedades da PBA, SE e BH (Norte) apresentam frequências superiores $(18 \%, 39 \%$ e $14 \%$, respectivamente) a do contorno $\mathrm{L}^{*}+\mathrm{H} \mathrm{L} \%(9 \%, 17 \%$ e $68 \%$, respectivamente). A Bahia mais uma vez atua como uma zona de transição entre Centro e Sul, com porcentagens superiores do contorno $\mathrm{L}^{*}+\mathrm{H} \mathrm{L} \%$. É interessante notar que nessa variedade o alinhamento adiantado do contorno LH apresenta a mesma especificação fonológica nuclear das variedades do Centro $\left(\mathrm{L}^{*}+\mathrm{H}\right)$.

\begin{tabular}{|l|r|r|r|}
\hline \multicolumn{4}{|c|}{ Norte } \\
\hline & PBA & \multicolumn{1}{|c|}{ SE } & Bh \\
\hline L* H\% & $73 \%$ & $44 \%$ & $0 \%$ \\
\hline L*+H H\% & $0 \%$ & $0 \%$ & $18 \%$ \\
\hline L* HL\% & $18 \%$ & $39 \%$ & $14 \%$ \\
\hline L*+H L\% & $9 \%$ & $17 \%$ & $68 \%$ \\
\hline
\end{tabular}

Tabela 2: Distribuição do contorno nuclear interrogativo no Norte.

\subsubsection{O contorno nuclear ascendente-descendente no Centro-Sul}

O contorno interrogativo ascendente-descendente (LHL), encontrado em todas as sete variedades analisadas, exibe dois tipos de formação fonológica. O primeiro tipo é formado por um acento nuclear ascendente $\mathrm{L}^{*}+\mathrm{H}$ e por uma fronteira baixa $\mathrm{L} \%$, predominante na Bh, RJ e MG. O segundo tipo é formado por um acento nuclear monotonal baixo $\mathrm{L}^{*}$ e por tom de fronteira complexo HL\%, predominante nas variedades do Sul (SC e RGS), e alternativo nas variedades do Norte (PBA e SE). 
O contorno nuclear $\mathrm{L}^{*}+\mathrm{H} \mathrm{L} \%$ nas variedades do $\mathrm{RJ}$ e de $\mathrm{MG}$ é formado por um tom baixo associado à cabeça do IP seguido de uma subida a partir da primeira metade da vogal tônica, alcançando o pico no início da postônica. Variações em termos de alinhamento foram observadas tanto entre informantes quanto entre variedades, sobretudo no padrão paroxítono final, em que há pouco espaço para concretizar os três tons que formam o contorno nuclear. Nesse contexto, MG apresenta um alinhamento adiantado em relação ao RJ, sendo caracterizado por um tom baixo alinhado à consoante e o tom alto alinhado entre o final da tônica e o início da postônica, formando assim uma vogal completamente ascendente (figura 5).

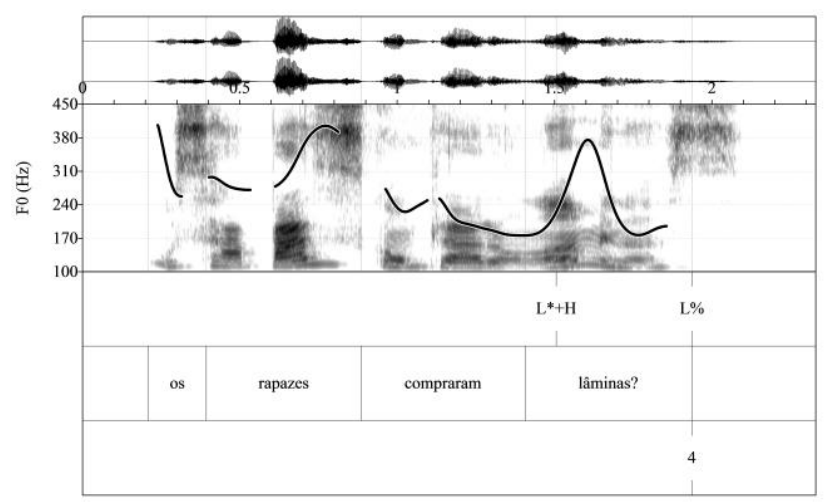

Figura 5: Enunciado interrogativo produzido pela informante LN de MG.

As variações no alinhamento dos tons $\mathrm{L}$ e $\mathrm{H}$ no núcleo tornam ambígua a associação tonal, que também poderia ser interpretada como um $\mathrm{L}+\mathrm{H}^{*} \mathrm{~L} \%$, tendo em vista dois fatores: 1) a subida ao longo da tônica e 2) a presença do tom alto alinhado com o final da tônica. A variação no alinhamento do pico do contorno ascendentedescendente nas variedades do Centro é tópico de discussão em estudos anteriores. Silva (2012) destaca a presença de uma maior quantidade de dados com alinhamento do pico no início da tônica nas variedades do sudeste, principalmente em MG. Em estudo recente, apenas a subida é tida como fonologicamente relevante na variedade do RJ (Frota\&Moraes, aceito. No presente corpus analisado, a opção pelo acento $\mathrm{L}^{*}+\mathrm{H}$ é justificada pelas seguintes razões: 1) a presença de um tom baixo necessariamente alinhado com a posição nuclear, seja no ataque, seja no núcleo; 2) as variações quanto ao tom alto (ora alinhado com tônica, ora com a postônica) o tornam mais instável que o tom baixo, caracterizando-o como um trailing tone (Grice, 1995); 3) a presença para todas as variedades de um tom nuclear baixo para as interrogativas, sendo essa a propriedade mais regular no que respeita este tipo frásico. A instabilidade do tom alto no contorno nuclear ascendente-descendente também é encontrada para o Italiano de Palermo (Grice, 1995). Segundo a autora, esta instabilidade ocorre em virtude da natureza do tom alto como trailing tone, o qual apresenta um comportamento mais instável por não estar diretamente associado a uma sílaba forte na cadeia métrica.

O contorno nuclear L* HL\% nas variedades de SC e RGS consiste em um tom completamente baixo, ancorado ao núcleo do IP, seguido por uma fronteira bitonal HL\%, que se realiza através de um movimento ascendente-descendente alinhado à(s) sílaba(s) postônicas, conforme observado no exemplo a seguir. 


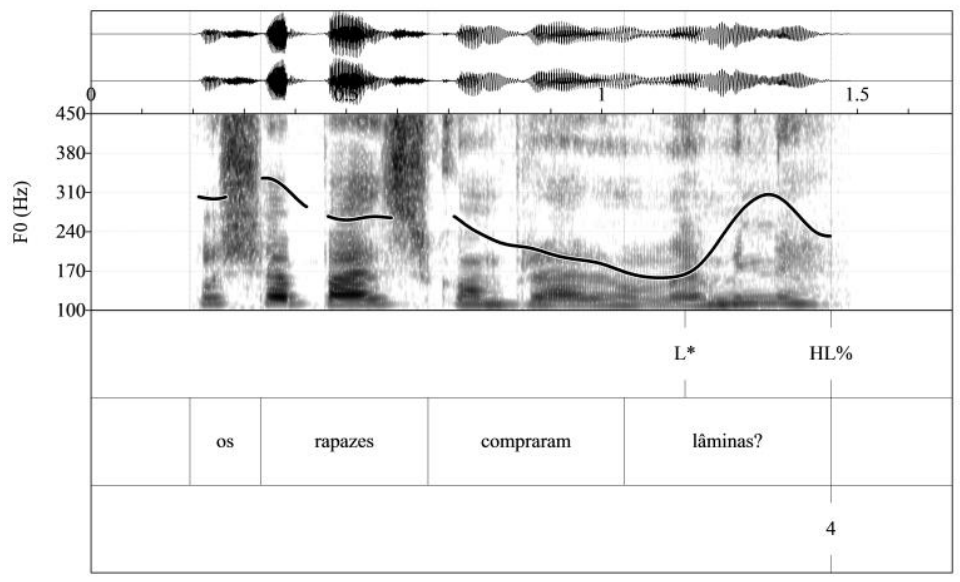

Figura 6: Enunciado interrogativo produzido pela informante ND de SC.

O comportamento entoacional do núcleo é irregular na região Sul. Observou-se existir a realização fonética de dois tipos de núcleos, um núcleo $\mathrm{L}^{*} \mathrm{HL} \%$, com subida iniciada na primeira postônica e o tom alto alinhado com a última postônica, conforme ilustrado acima; e um segundo tipo de contorno LHL, no qual o movimento ascendente ainda se inicia na tônica e o tom alto se alinha à primeira postônica, semelhante ao núcleo $\mathrm{L}^{*}+\mathrm{H} \mathrm{L} \%$ do Centro. Esse segundo tipo de comportamento resulta de uma associação secundária do tom alto de fronteira ao núcleo do IP, pelo que se adotou a anotação $\mathrm{L}^{*}(+\mathrm{H}) \mathrm{HL} \%$. A realização fonética do contorno nuclear $\mathrm{L}^{*}+\mathrm{H} \mathrm{L} \%$ do Centro apresenta características diferentes do contorno $\mathrm{L}^{*}(+\mathrm{H}) \mathrm{HL} \%$ do Sul, uma vez que (i) o tom baixo nuclear do Sul ocupa metade da vogal tônica, ao passo que no Centro esse mesmo tom realiza-se na consoante ou no início da vogal tónica, exibindo um alinhamento mais adiantado em relação às variedades do Sul e (ii) o alinhamento do tom alto nos casos de $\mathrm{L}^{*}(+\mathrm{H}) \mathrm{HL} \%$ do Sul geralmente ocorre na primeira vogal postônica, diferenciando-se, assim, do comportamento $\mathrm{L}^{*}+\mathrm{H} \mathrm{L} \%$ do Centro, no qual o tom alto encontra-se geralmente alinhado com a consoante da primeira postônica. A instabilidade do tom alto na configuração interrogativa LHL também foi encontrada nas línguas do leste europeu, como o Húngaro (Grice, 2000), que são explicadas por diferentes regras de associação do phrase accent à sílaba acentuada.

Abaixo são apresentadas as tabelas com as porcentagens dos contornos interrogativos observados na região Centro e Sul. Embora tenha sido encontrado o mesmo tipo de contorno, as variações no alinhamento dos tons à fiada segmental indicam diferenças fonológicas entre as duas regiões. Observa-se que a região Centro apresenta um comportamento bastante consistente, com uma produção categórica do contorno $\mathrm{L}^{*}+\mathrm{H} \mathrm{L} \%$. A região Sul, por outro lado, apresenta um comportamento variável em termos de alinhamento (assim como observado no contorno ascendente na região Norte), exibindo o contorno L* HL\% como predominante nas variedades de SC e RGS (46\% e $75 \%$, respectivamente), com a possibilidade de receber associação do tom de fronteira ao núcleo do IP L*(+H) HL\% (30\% e 23\%, respectivamente). 


\begin{tabular}{|l|r|r|}
\hline \multicolumn{3}{|c|}{ Centro } \\
\hline & MG & RJ \\
\hline L* H\% & $0 \%$ & $0 \%$ \\
\hline L*+H H\% & $0 \%$ & $0 \%$ \\
\hline L* HL\% & $0 \%$ & $0 \%$ \\
\hline L*+H L\% & $98 \%$ & $100 \%$ \\
\hline
\end{tabular}

\begin{tabular}{|l|r|r|}
\hline \multicolumn{3}{|c|}{ Sul } \\
\hline & SC & RGS \\
\hline L* H\% $^{*}$ & $1 \%$ & $0 \%$ \\
\hline L*+H H$\%^{*}$ & $0 \%$ & $0 \%$ \\
\hline L* HL\% $^{*}$ & $46 \%$ & $75 \%$ \\
\hline $\begin{array}{l}\text { L*(+H) } \\
\text { HL\% }\end{array}$ & $30 \%$ & $23 \%$ \\
\hline
\end{tabular}

Tabela 3: Distribuição do contorno nuclear interrogativo no Centro e Sul.

Em todas as regiões foi observado o truncamento do tom baixo na fronteira como estratégia de acomodação tonal em contextos com tônica final à semelhança do que ocorre nas variedades do sul da Itália (Grice, 2005). As diferenças no alongamento do tom baixo na sílaba tônica revelam pistas para formação fonológica do contorno nuclear, conforme pode ser observado nas figuras abaixo. Nas variedades do Centro (figura 7, painel esquerdo), observa-se que a realização do núcleo $\mathrm{L}^{*}+\mathrm{H}$ se dá através do contorno ascendente final, que ocupa a maior parte da sílaba. Nos casos das variedades do Sul (figura 7, painel direito), o espaço da sílaba tônica é dividido para realização do núcleo $L^{*}$ e de parte da fronteira $\mathrm{H}$. Nesse caso, o tom baixo estende-se até o meio da sílaba, sendo seguido de um contorno ascendente final.
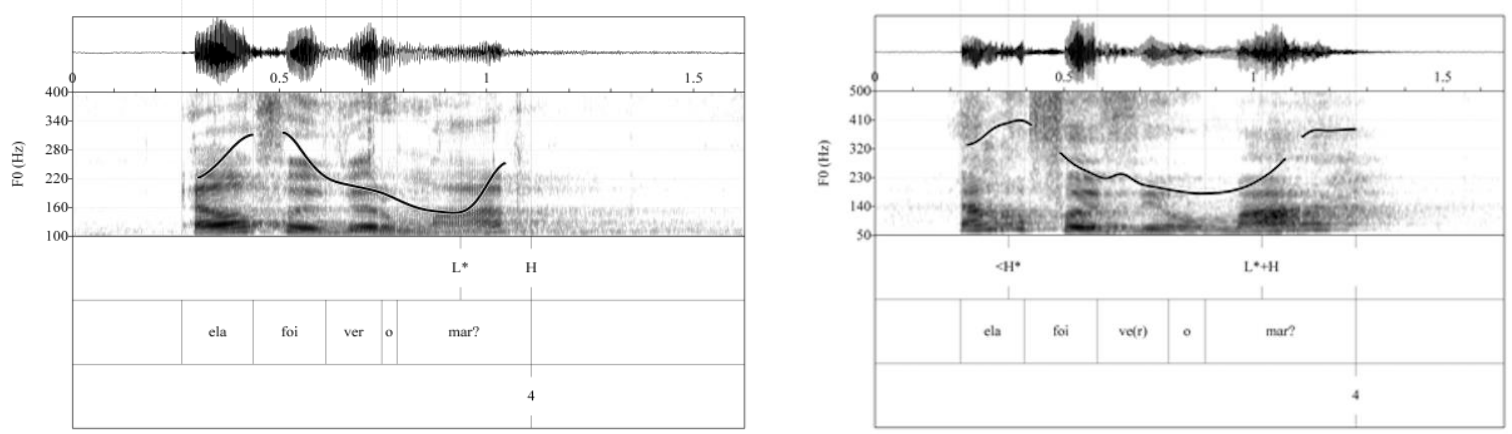

Figura 7: Enunciados interrogativos produzidos pelas informantes do RJ (painel direito) e RGS (painel esquerdo).

$\mathrm{Na}$ variedade de Santa Catarina, no entanto foram encontradas outras estratégias de acomodação tonal. Apesar de a estratégia de truncamento ter sido predominante, foram encontradas para as quatro informantes estratégias de compressão dos tons, que são consistentes com os resultados de estudos prévios nessa região (Nunes, 2011). As diferenças de associação tonal observadas entre as regiões conduziram a uma análise estatística a fim de verificar o alinhamento dos tons. A hipótese levantada a partir da observação acústica dos dados é a de que o alinhamento do tom alto será mais tardio nas regiões Norte e Sul, uma vez que, em ambas as localidades, esse tom é fonologicamente especificado como fronteira do IP. Por outro lado, é esperado um alinhamento mais adiantado no Centro, uma vez que o tom alto nessa região é fonologicamente especificado como núcleo do IP. Foram medidas as distâncias temporais do tom L em relação ao onset da tônica e do tom $\mathrm{H}$ em relação ao offset da tônica. Foram aplicados dois testes estatísticos para análise do alinhamento nas interrogativas. Para o alinhamento do tom alto, foi empregado o teste $\mathrm{T}$, comparando-se aos pares as regiões 124 
Norte, Centro e Sul. Para o alinhamento do tom baixo, foi empregado o teste não paramétrico Mann Whitney, uma vez que os resultados dos testes Kolmogorov-Smirnov e Shapiro-Wilk revelam que a distribuição dos dados não segue uma distribuição normal. Os testes confirmam as hipóteses apresentadas acima. Em relação ao alinhamento do tom alto, o Norte é diferente do Centro $(\mathrm{F}=6,518, \mathrm{p}<0,05)$ e do Sul $(\mathrm{t}=3,042, \mathrm{p}<0,05)$, assim como o Centro é diferente do $\mathrm{Sul}(\mathrm{F}=7,068, \mathrm{p}<0,05)$. Em relação ao alinhamento do tom baixo, o Norte é diferente do Centro $(Z=-5.176, p<$ $0,05)$ e do $\operatorname{Sul}(Z=-3,466, p<0,05)$, assim como o Centro é diferente do Sul $(Z=-$ $2,434, p<0,05)$. No gráfico abaixo são visualizadas as diferenças no alinhamento dos tons $\mathrm{L}$ e $\mathrm{H}$ entre as regiões.

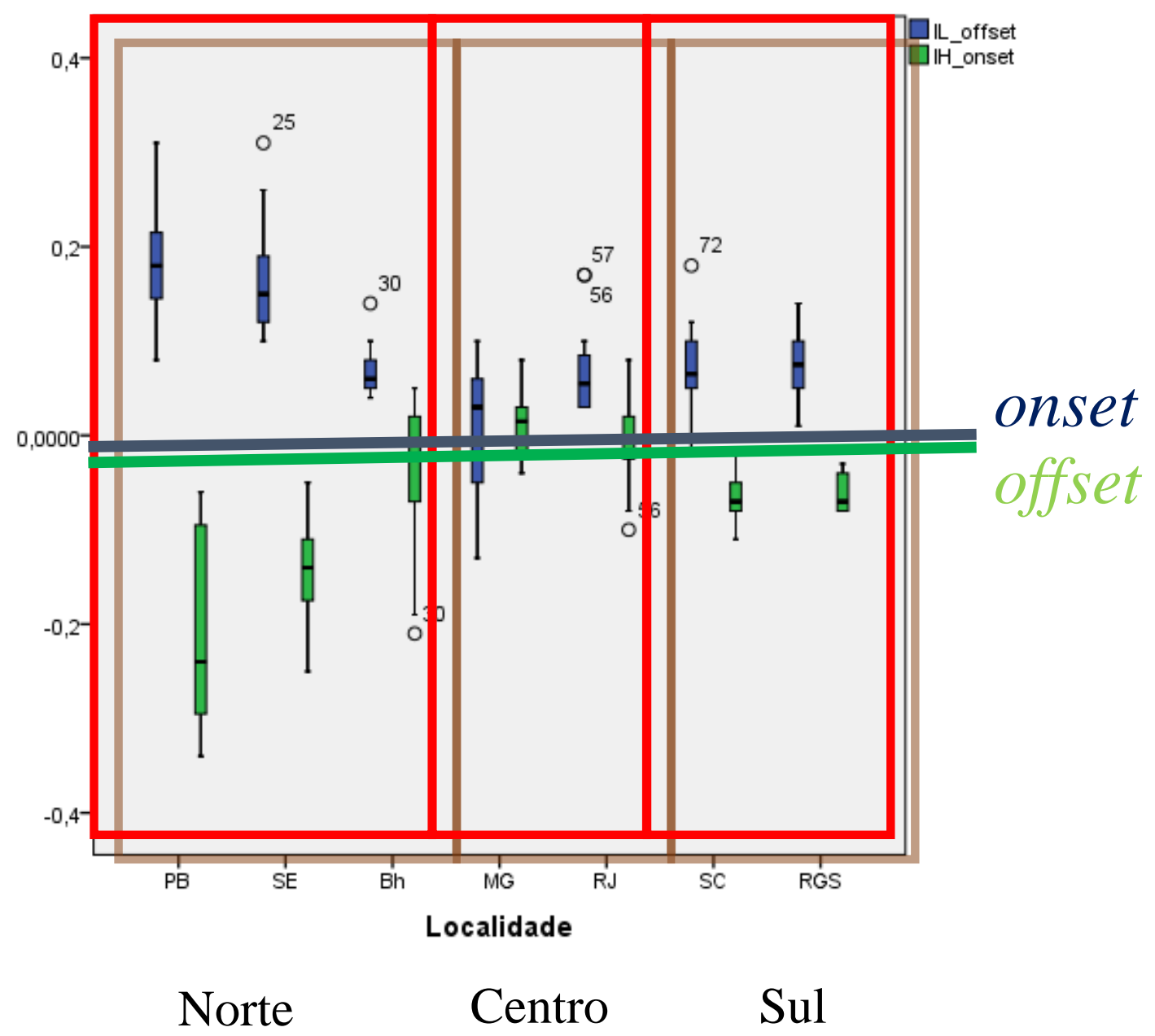

Figura 20: Medidas do alinhamento dos tons nucleares da configuração interrogativa LHL.

Segundo Ladd (2008), o alinhamento pode consistir em diferenças fonéticas ou graduais, tal como foi mostrado para as declarativas, como também em diferenças fonológicas ou categóricas, como a presente análise estatística mostra para as interrogativas no PB. No Norte, os núcleos L* H\%, L*+H H\% e L* HL\% apresentam o tom $\mathrm{H}$ associado à fronteira que, por conseguinte, exibe um alinhamento temporal mais 
tardio, observado através de uma maior distância no alinhamento dos tons. No Centro, a associação do tom $\mathrm{H}$ ao núcleo $\left(\mathrm{L}^{*}+\mathrm{H} \mathrm{L} \%\right)$ desencadeia um alinhamento do tom alto mais adiantado e mais próximo do tom baixo do núcleo. $\mathrm{O}$ Sul, à semelhança da região Norte, apresenta o tom alto associado à fronteira ( $\left.\mathrm{L}^{*} \mathrm{HL} \%\right)$; no entanto a associação secundária do tom alto ao núcleo $\left(\mathrm{L}^{*}(+\mathrm{H}) \mathrm{L} \%\right)$ explica o fato de o alinhamento dos tons L e H nessa região se apresentar como intermédio entre as regiões Norte e Centro, verificando-se o fato de o tom $\mathrm{H}$ ocupar tanto o espaço do núcleo quanto o da fronteira do IP.

\section{Análise fonológica do contorno nuclear declarativo e interrogativo no PB}

A diferença entre declarativas e interrogativas totais no Português é unicamente marcada pela entoação. As variações observadas entre as regiões indicam a existência de diferentes mecanismos fonológicos que marcam na gramática a distinção entre os dois tipos frásicos. Tendo isso em vista, os objetivos dessa secção são (1) representar fonologicamente os contornos nucleares de declarativas e interrogativas no $\mathrm{PB}$, com base nas propriedades apresentadas na seção anterior; (2) discutir os recursos entoacionais que concorrem para estabelecer a oposição fonológica entre declarativas e interrogativas neutras no PB, tendo em conta a formação do núcleo do IP.

$\mathrm{O}$ contorno nuclear declarativo é formado por um núcleo bitonal $\mathrm{H}+\mathrm{L} *$ e por uma fronteira monotonal $\mathrm{L} \%$. As principais propriedades fonológicas que formam esse contorno descendente são: (1) o alinhamento sistemático do tom alto à sílaba que precede imediatamente a tônica, independentemente da separação entre esse constituinte e a fronteira inicial da palavra prosódica nuclear; (2) a presença de um tom baixo $\mathrm{L}^{*}$, sendo um tom característico porque atinge a linha de base de F0 das informantes e está sistematicamente alinhado com a tônica, tanto em contextos segmentais estreitos com sílaba final acentuada quanto em contextos com cadeia segmental estendida, como em acento antepenúltimo.

A produção das interrogativas totais, por outro lado, revela mais diferenças do que semelhanças fonológicas entre os dialetos. O principal traço comum entre os dialetos é o núcleo, invariavelmente formado pela associação de um tom baixo $\mathrm{L}^{*}$, o qual pode ser monotonal $\mathrm{L}^{*}$ ou bitonal $\mathrm{L}^{*}+\mathrm{H}$, sendo neste caso formado por um tom nuclear baixo seguido de um trailing tone alto. Há também a presença de contornos ascendentes em todas as variedades, que podem ou não ser seguidos de uma descida ao final do enunciado. Há maior variação entre os dialetos no que tange aos tipos de tons disponíveis para se associarem à fronteira (L\%, H\% e HL\%). A combinação entre os dois núcleos e as três fronteiras resulta nos seguintes acentos: $\mathrm{L}^{*} \mathrm{H} \%, \mathrm{~L}^{*}+\mathrm{H} \mathrm{H} \%, \mathrm{~L}^{*}$ $\mathrm{HL} \%, \mathrm{~L} *+\mathrm{H} \mathrm{L} \%$.

Os contornos nucleares ascendentes $\mathrm{L}^{*} \mathrm{H} \%$ (PBA e SE) e $\mathrm{L}^{*}+\mathrm{H} \mathrm{H} \%$ (Bh) diferem entre si em termos de alinhamento: um tom completamente baixo na sílaba nuclear, o que não ocorre no segundo caso. Em vez disso, o núcleo $\mathrm{L}^{*}+\mathrm{H}$ realiza-se através de um contorno ascendente na sílaba nuclear. Ambos os comportamentos não são afetados pela posição do acento nem pela distância da fronteira inicial da última palavra prosódica.

Conclui-se, através da observação das propriedades dos contornos nucleares do Centro e do Sul, que o alinhamento do tom alto funciona para as interrogativas como 
uma propriedade fonológica que diferencia dois tipos de contornos nucleares, os quais apresentam comportamentos sistematicamente distintos. É interessante notar ainda que o estatuto fonológico do tom alto nos dois contornos nucleares se reflete na variabilidade temporal desse tom, observada na superfície dos enunciados.

As propriedades quanto à associação e alinhamento dos tons fornecem pistas acerca da marca gramatical que contribui para o significado de interrogatividade no PB. No Centro, essa oposição consiste na associação de diferentes entidades fonológicas ao núcleo do IP, sendo o núcleo descendente $\mathrm{H}+\mathrm{L}^{*}$ nas declarativas e ascendente $\mathrm{L}^{*}+\mathrm{H}$ nas interrogativas, acompanhados de fronteira baixa L\%, o que confirma os resultados de estudos anteriores (Frota\&Moraes, aceite).
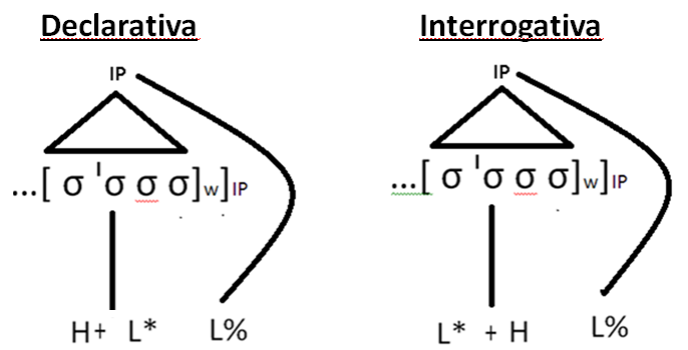
Centro.

Figura 8: Representação fonológica dos contornos declarativo e interrogativo no

Nas variedades do Norte e do Sul, há a combinação de diferentes entidades que opõem fonologicamente declarativas e interrogativas. Nas variedades da Paraíba e de Sergipe (Norte), o núcleo descendente nas declarativas opõe-se ao tom baixo nas interrogativas e a fronteira baixa nas declarativas opõe-se à fronteira alta nas interrogativas.
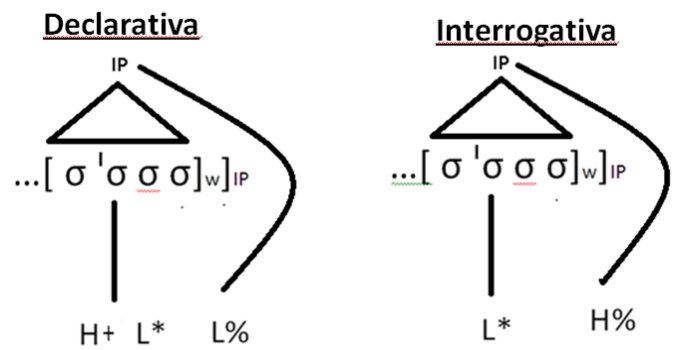
Norte.

Figura 9: Representação fonológica dos contornos declarativo e interrogativo no

Nas variedades de Santa Catarina e do Rio Grande do Sul (Sul), o núcleo descendente nas declarativas opõe-se ao tom baixo nas interrogativas e a fronteira baixa nas declarativas opõe-se à fronteira descendente nas interrogativas. 

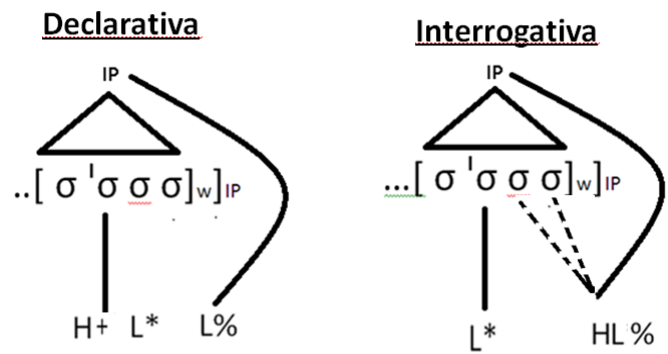

Figura 10: Representação fonológica dos contornos declarativo e interrogativo no Sul.

Os resultados do presente estudo mostram que, para o PB, a seleção de ambas as entidades que formam o contorno nuclear são empregadas na gramática para estabelecer a oposição entre os tipos frásicos. A combinação do núcleo e da fronteira para estabelecer a oposição fonológica entre declarativas e interrogativas é o que há de mais comum tanto para os dialetos do PB quanto para os dialetos do PE. Apesar de toda a possível variação observada a partir dos dados, é importante considerar sobretudo a regra mais geral, que determina um movimento melódico de descida nas declarativas e de subida nas interrogativas, os quais podem ser fonologizados independentemente do seu alinhamento à fiada métrica.

\section{Conclusões}

A análise fonológica e dialetal dos dados apontam para um comportamento entoacional equilibrado. A associação fonológica dos tons nos enunciados declarativos e interrogativos revela que existem mais semelhanças do que diferenças entre as variedades do $\mathrm{PB}$ : (1) o contorno $\mathrm{H}+\mathrm{L}^{*} \mathrm{~L} \%$ nas declarativas é comum a todas as variedades; (2) a associação tom $\mathrm{L}^{*}$ ao núcleo das interrogativas também é comum a todas as variedades, sendo especificado como um tom monotonal $\mathrm{L}^{*}$ ou bitonal $\mathrm{L}^{*}+\mathrm{H}$; (3) a frequência dos dados indica que o contorno ascendente-descendente está a difundir-se para o Norte, embora não seja o dominante; (4) o truncamento é a estratégia de acomodação tonal mais comum entre as regiões.

Os resultados indicam a formação de um continuum linguístico, verificado no alinhamento nuclear das declarativas - Norte $(\mathrm{H}+) \mathrm{L}^{*}$ e Centro-Sul $\mathrm{H}+\mathrm{L}^{*}$-, bem como na configuração nuclear das interrogativas - Norte (LH), Centro-Sul (LHL). Apesar de toda a variação na especificação fonológica, o contorno descendente declarativo e o contorno ascendente-descendente interrogativo aparecem em todas as variedades analisadas. Esse arranjo espacial pode revelar uma tendência fonológica mais geral do sistema, fixada no contorno nuclear do IP, que consiste em um contorno descendente para expressar a assertividade e um contorno ascendente na sílaba nuclear do IP para expressar a interrogatividade, estabelecendo desse modo uma oposição fonológica uniforme entre declarativas e interrogativas neutras no PB. Nossa intenção é testar essa hipótese através de estudos de percepção em trabalhos futuros (Silva, em curso).

Agradecimentos

A todos os informantes envolvidos, à toda equipe do projeto InAPoP, em especial, à Verónica Crespo-Sendra, pela revisão dos dados e à Marisa Cruz, pelos comentários; aos pesquisadores brasileiros Aline Fonseca, Carolina Serra, Gisela Collischonn, João 
Moraes, Pedro Felipe, Silvana Ribeiro e Vanessa Nunes pelo auxílio que prestaram ao longo da recolha dos dados. À Coordenação de Aperfeiçoamento de Pessoal de Nível Superior - CAPES pela bolsa de doutoramento pleno no exterior de Joelma Castelo $\left(\mathrm{n}^{\circ}\right.$ 094912-4). A presente pesquisa foi desenvolvida no âmbito do InAPoP project (PTDC/CLE-LIN/119787/2010), financiado pela Fundação para a Ciência e a Tecnologia/MCTES (PIDDAC).

\section{Referências}

Atterer, M. \& D. R. Ladd. (2004) On the phonetics and phonology of "segmental anchoring' of F0: evidence from German. Journal of Phonetics, 32, pp. 177-197

Barbiers, S. (2008) Locus and limits of syntactic microvariation. Lingua 119, pp. 16071623

Boersma, P., D. Weenink. (2010). Praat - doing phonetics by computer. Versão 5.3.45

Britain, D. (2007) Space and Spatial Diffusion. In: The handbook of language variation and change. Chambers, J. K., Peter Trudgill and Natalie-Estes (eds). Blackwell Publishing, 2003. Blackwell Reference Online. 31 December 2007 http://www.blackwellreference.com/subscriber/tocnode?id=g9781405116923_chunk g978140511692333

Crespo-Sendra, V., M. Cruz, J. Silva \& S. Frota. (2014) Asking questions across Portuguese varieties. Talk presented at the 6thInternational Conference on Tone and Intonation in Europe (TIE). Netherlands: University of Utrecht.

Cunha, C. (2000). Entoação regional noPortuguês do Brasil. Tese de Doutoramento em Língua Portuguesa. Rio de Janeiro, Faculdade de Letras, UFRJ.

Frota, S. \& J. Moraes (aceito) Intonation of European and Brazilian Portuguese. In W. L. Wetzels, S. Menuzzi \& J. Costa. (eds.) The Handbook of Portuguese Linguistics. Wiley

Frota, S. \& P. Prieto. (2015). Intonation in Romance: systemic similarities and differences. In S. Frota \& P. Prieto (eds). Intonation in Romance. Oxford: Oxford University Press.

Frota, S. (2000) Focus and prosody in European Portuguese. Phonological phrase and intonation. New York: Garland Publishing.

Frota, S. (2002) Nuclear falls and rises in European Portuguese: a phonological analysis of declarative and question intonation. Probus 14, pp. 113-146. 
Frota, S. (2014) The intonational phonology of European Portuguese. In Sun-Ah Jun (ed.). Prosodic Typology II. Oxford: Oxford University Press, pp. 6-42

Frota, S. (2014) The intonational phonology of European Portuguese. In Sun-Ah Jun (ed.). Prosodic Typology II. Oxford: Oxford University Press, pp. 6-42

Frota, S. (Coord). 2012-2014. Interactive Atlas of the Prosody of Portuguese Project (Webplatform available at http://www.fl.ul.pt/LaboratorioFonetica/InAPoP/).

Frota, S. (submetido) Surface and structure: transcribing intonation whithin and across languages. Laboratory Phonology.

Frota, S., M. Cruz., F. Fernandes-Svartman, G. Collischonn, A. Fonseca, C. Serra, P. Oliveira \& M. Vigário (2015) Intonational variation in Portuguese: European and Brazilian varieties. In S. Frota \& P. Prieto (eds). Intonation in Romance. Oxford: Oxford University Press.

Grabe, E. (2004) Intonational variation in urban dialects of English spoken in the British Isles. In: PETER, G.; PETERS, J. (Ed.) Regional variation in intonation. Tübingen: Niemeyer, pp.9-32.

Grice, M, M. D' Império, M. Savino, C. Avesani. (2005). Strategies for Intonation Labelling across Varieties of Italian. In: Sun-Ah Jun. Prosodic Typology - The Phonology of Intonation and Phrasing. Sun-Ah Jun (ed.). Oxford: Oxford University Press, pp. 362- 389.

Grice, M. (1995) The intonation of interrogation in Palermo Italian: Implications for intonation theory. Tubingen: Niemeyer.

Grice, M., R. Ladd, A. Arvanite (2000) On the place of the phrasal accents in intonational phonology, Phonology 17. Cambridge: Cambridge University Press, pp. $143-185$.

Gussenhoven, C. (2004). The phonology of tone and intonation. Cambridge: Cambridge University.

Haan, J. (2002) Speaking of Questions. An Exploration of Dutch Question Intonation. LOT. Dissertation Series, $\mathrm{Nr}$ 52, Netherlands Graduate School of Linguistics, Utrecht

Ladd, R. (2008) Intonational Phonology. $2^{\mathrm{a}}$ ed. Cambridge: Cambridge University Press. 
Lobo, T. (2003). A questão da periodização da história linguística do Brasil. In: Castro, I. \&Duarte, I. Razões e emoções: miscelânea de estudos em homenagem a Maria Helena Mira Mateus. Vol.1. Lisboa: Imprensa Nacional - Casa da Moeda.

Mateus, M.; Brito, A.; Duarte, I.; Faria, I.; Frota, S.; Matos, G. Oliveira, F. ;Vigário, M.; Villalva, A. (2003) Gramática da Língua Portuguesa (7ª edição). Lisboa: Editora Caminho

Moraes, J. (2008) The Pitch Accents in Brazilian Portuguese: analysis by synthesis. In: Proceedings of the Speech Prosody. Campinas: Unicamp.

Nascentes, A. (1953) O linguajar carioca. Rio de Janeiro: Organização Simões.

Nunes, V. (2011) Análises entonacionais de sentenças declarativas e interrogativas totais nos falares florianopolitano e lageano. Dissertação de metrado em Linguística. Florianópolis, UFSC.

Obanauer, H. (2006) Special Interrogatives - Left Periphery, Wh-Doubling, and (Apparently) Optional Elements, Jenny Doetjes and Paz Gonzalves, eds., Romance Languages and Linguistic Theory 2004 - Selected Papers from 'Going Romance 2004'. Amsterdam/Philadelphia: John Benjamins, pp. 247-273

Prieto, P. \& P. Roseano (eds.). (2010). Transcription of Intonation of the Spanish Language. Lincom Europa: München.

Savino, M. 2012. The intonation of polar questions in Italian: where is the rise? Journal of the International Phonetic Association, vol. 42, 23-48.

Silva, J. (2012). O alinhamento do pico da F0 na questão total da região sudeste: um estudo preliminar. Pôster apresentado no VII GSCP International Conference: Speech And Corpora. Universidade Federal de Minas Gerais, Belo Horizonte.

Silva, J. em curso. A entoação dos Enunciados Assertivos e Interrogativos nos Falares das Regiões Nordeste, Sudeste e Sul do Brasil. PhD in Linguistics, University of Lisbon. Supervisor: Sónia Frota.

Silvestre, A. (2012) A entoação regional dos enunciados assertivos nos falares das capitais brasileiras. Dissertação de mestrado em Língua Portuguesa. Rio de Janeiro, Faculdade de Letras, UFRJ. 